\title{
Does the get up and go test improve predictive accuracy of the Triage Risk Screening Tool or Rowland questionnaire in older patients admitted to the emergency department? Els Devriendtt ${ }^{a, d}$, Mieke Deschodt ${ }^{a, d, f}$, Maarten Delaere ${ }^{\mathrm{a}}$, Johan Flamaing ${ }^{\mathrm{b}, \mathrm{d}}$, Marc Sabbe ${ }^{\mathrm{c}, \mathrm{e}}$ and Koen Milisen ${ }^{\mathrm{a}, \mathrm{d}}$
}

\begin{abstract}
Objectives To assess the diagnostic characteristics of the get up and go test (GUGT) as a stand-alone test and in combination with the Flemish Triage Risk Screening Tool (fTRST) and Rowland questionnaire. One aim was to determine whether the diagnostic accuracy of these instruments could be improved for predicting unplanned emergency department (ED) readmission following ED discharge.
\end{abstract}

Methods We carried out a prospective cohort study at the ED of the University Hospitals Leuven, Belgium. All patients aged at least 75 years $(n=380)$ completed fTRST, Rowland, and GUGT testing at the index ED admission. Diagnostic characteristics for unplanned ED readmission were determined for hospitalized and discharged patients 1 and 3 months after the index ED visit.

Results In both hospitalized and discharged patients, fTRST and Rowland (cut-off $\geq 2$ ) had good to excellent sensitivity and negative predictive value (NPV) but low to moderate specificity and accuracy; GUGT had low sensitivity and good to excellent NPV and specificity. The combined fTRST/GUGT or Rowland/GUGT had moderate to excellent NPV (56.3-94.3\%). The combined fTRST (cut-off $\geq 2$ )/GUGT had low sensitivity and moderate to excellent specificity. Sensitivity of the combined Rowland (cut-off $\geq 4$ )/GUGT was good at the 1-month follow-up and moderate at the 3-month follow-up for hospitalized

\section{Introduction}

About $12-21 \%$ of all patients admitted to the emergency department (ED) are 75 years or older, and this percentage is expected to increase in the coming years [1]. Older ED patients have more urgent problems, longer ED stays, higher admission rates, and more adverse health outcomes after discharge (e.g. readmission, functional decline, institutionalization, mortality) compared with younger patients $[1,2]$. One-half to three-quarters of all older patients are hospitalized after ED discharge, indicating that a considerable proportion is discharged to their residence $[1,3,4]$. Unfortunately, $12-20 \%$ of these patients are readmitted 1 month after being discharged,

Supplemental digital content is available for this article. Direct URL citations appear in the printed text and are provided in the HTML and PDF versions of this article on the journal's website (www.euro-emergencymed.com). patients; it was low for discharged patients. Specificity was low for hospitalized patients and good for discharged patients.

Conclusion Neither the objective measure of mobility (GUGT) nor the combined fTRST/GUGT or Rowland/GUGT improved the results. Our analysis shows that the predictive accuracy of the stand-alone, self-reported screening instruments fTRST and Rowland (cut-off $=2$ ) is still good. This study also confirmed their previously known limitations. European Journal of Emergency Medicine 25:46-52 Copyright (c) 2018 Wolters Kluwer Health, Inc. All rights reserved.

European Journal of Emergency Medicine 2018, 25:46-52

Keywords: emergency department, geriatric, hospitalization, screening, unplanned readmission

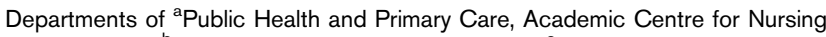
and Midwifery, ${ }^{b}$ Clinical and Experimental Medicine, ${ }^{c}$ Public Health and Primary Care, Emergency Medicine, KU Leuven - University of Leuven, Departments of ${ }^{\mathrm{d}}$ Geriatric Medicine, ${ }^{e}$ Emergency Medicine, KU Leuven - University of Leuven, University Hospitals Leuven, Belgium and ${ }^{f}$ Department of Public Health, Institute of Nursing Science, University of Basel, Basel, Switzerland

Correspondence to Koen Milisen, PhD, RN, Department of Public Health and Primary Care, Academic Centre for Nursing and Midwifery, KU Leuven University of Leuven, Kapucijnenvoer 35/4, 3000 Leuven, Belgium Tel: + 32163779 79; fax: +32 163369 70; e-mail: koen.milisen@kuleuven.be

Received 12 December 2015 Accepted 6 June 2016

$19-24 \%$ at 3 months, and up to $40 \%$ at 6 months after discharge [4-7].

Comprehensive geriatric assessment (CGA) is effective in detecting geriatric care problems and addressing the complex and specific needs of frail older adults [8]. Evidence shows that CGA-based interventions in the ED improve the detection of common geriatric problems and may therefore help prevent unplanned readmissions [9-13]. However, time constraints, caseload, lack of geriatric expertise, and limited healthcare resources hinder the widespread use of CGA in the ED. The widespread use of accurate and simple case-finding or screening tools could be a critical first step in identifying those patients who would benefit most from CGA-based interventions [14].

DOI: 10.1097/MEJ.0000000000000413 
The Flemish version of the Triage Risk Screening Tool (fTRST) and Rowland questionnaire are short and easyto-administer screening tools based on self-reported measures. They have been evaluated for their efficacy in predicting unplanned readmission after an ED visit [6,15-17]. In a previous study, we obtained moderately robust results for the fTRST, but with low specificity [6]. In this same study, we obtained somewhat better results for the Rowland questionnaire in predicting unplanned ED readmission (i.e. fewer false-positive detections in patients immediately discharged from the ED). Consistent with these findings, a recent meta-analysis concluded that instruments based on self-reported measures still fail to distinguish between patients at high or low risk for adverse events [18]. Carpenter et al. [18] recommended that researchers study the predictive value of other brief instruments not yet tested in the ED and evaluate objective measures such as mobility in addition to self-reported measures.

A frequently used objective measure for mobility assessment is the get up and go test (GUGT), which examines the ability of patients to stand up from a sitting position and walk independently [19]. As it requires minimal equipment, the GUGT can easily be administered in the ED. To our knowledge, only one study has evaluated the GUGT as a predictor of ED readmission, but this study only examined the correlation between GUGT results obtained during the ED visit and the number of days to ED readmission [20]. Hence, in the present study, we aimed to determine the predictive accuracy of the GUGT as a stand-alone test and in combination with the fTRST and Rowland questionnaire in predicting unplanned $\mathrm{ED}$ readmissions following ED discharge.

\section{Methods}

The methodology used in the current study has been described in detail elsewhere [4]. A brief summary is presented below.

A prospective cohort study was carried out between November 2011 and February 2012 at the ED of the University Hospitals Leuven, Leuven, Belgium [4]. Patients were included if they were 75 years or older, living at home, and Dutch speaking. We excluded nursing home residents, patients transferred to the ED from a hospital ward or another hospital, and patients intubated or suffering from a medical condition that prevented or hindered them from participating in an interview. The study was approved by the Medical Ethics Committee of the University Hospitals Leuven (B 3222011112405). Informed (proxy) consent was obtained for all study patients.

\section{Variables and measurements}

Data on age, sex, living situation, and instrumental activities of daily living (i.e. nursing care, home care, meals on wheels, cleaning help, shopping assistance) were collected from each participant. We also collected the following clinical data: ED priority level of the participant Emergency Severity Index [21]; the main reason for ED admission; comorbidity Cumulative Illness Rating Scale [22]; delirium status (confusion assessment method) [23]; cognitive status (sweet 16) [24]; functional status (Katz Index) [25]; and number of falls in the last year.

\section{Primary outcome}

The primary outcome variable of the study was 'unplanned ED readmission'; it was defined as a subsequent or repeat ED visit that followed the index ED visit or hospitalization, and could not have been foreseen at the time of ED or hospital discharge [26].

\section{Screening instruments}

Details of the screening instruments are summarized in Supplementary data 1, Supplemental digital content 1, http://links.lww.com/EJEM/A126

The fTRST comprises five dichotomous questions (yes/ no answers) and was designed for identification of older ED patients at risk for ED readmission, hospitalization, or institutionalization $[7,16]$. The tool includes cognitive impairment, living alone or in the absence of a central caregiver, difficulties with walking or transfer, admission to ED during the last month, or hospitalization within the last 3 months and polypharmacy. Patients who had cognitive impairment or who answered 'yes' on at least two other questions were considered to be 'at risk'.

The Rowland et al. [27] questionnaire is a seven-item questionnaire (yes/no answers) developed to identify patients at risk for readmission. The tool includes the use of walking aids or assistance when walking or transferring, assistance in dressing, dependency for collecting pension, dependency for shopping, use of a daycare center, use of meals on wheels, and the presence of home help. A patient was considered to be at risk if he or she answered 'yes' on at least four items [27].

The GUGT evaluates the patient's ability to stand up from a chair, walk $3 \mathrm{~m}$, turn around, walk back, and sit on the chair again [19]. A patient received a score of 0 if he or she needed help from another individual to complete the test. The patient received a score of 1 if the test could be completed independently, with or without a walking aid.

\section{Procedure}

Baseline data were collected at the index ED visit by trained research nurses immediately after the triage and the first medical assessment. For patients discharged from the ED to their residence, data on unplanned ED readmission were collected 1 and 3 months after discharge by contacting the patients or relatives by telephone. For hospitalized patients, data on unplanned ED 
readmission 1 and 3 months after hospital discharge were collected on the basis of chart review.

\section{Analysis}

Means and SD were calculated for continuous variables, whereas absolute numbers and percentages were reported for categorical variables. Prediction of unplanned ED readmission was studied in relation to two types of patients: 'hospitalized patients' and 'discharged patients'. The latter type were patients who were not subsequently hospitalized after the index ED visit. We calculated the sensitivity, specificity, negative predictive value (NPV), positive predictive value (PPV), accuracy, likelihood ratio of positive test $(\mathrm{LH}+)$, likelihood ratio of negative test $(\mathrm{LH}-)$, and area under the receiver operating characteristic curve (AUC) for unplanned ED readmission at 1 and 3 months after the index ED visit for the three screening instruments when used as a stand-alone test and when used in combination. The predictive accuracy of the fTRST using cut-off values of 1,2 , and 3 , and that of the Rowland questionnaire using cut-off values of 2,3, and 4 were evaluated for the stand-alone test. The twostep approach was used to evaluate the predictive accuracy of the combination of the fTRST (cut-off values of $1,2,3$ ) or the Rowland (cut-off values of $2,3,4$ ) (step 1), followed by the GUGT (able or unable to execute the GUGT) (step 2). Predictive accuracy scores were categorized as low (0-49); moderate (50-69); good (70-89); and excellent (90-100). All analyses were carried out using SPSS 20.0 (2011, IBM SPSS Statistics for Windows, version 20.0; IBM Corp., Armonk, New York, USA).

\section{Results}

\section{Patient characteristics}

Of the 380 eligible patients, 283 (74.5\%) were hospitalized and $97(25.5 \%)$ were immediately discharged (Fig. 1). The mean age for all 380 patients was 83.1 years, and $58.9 \%$ were women. Most patients were living with a relative $(n=225,59.2 \%)$. Additional characteristics are summarized in Table 1.

Of the hospitalized patients, 26 (9.2\%) and 59 (20.8\%) had an unplanned ED readmission within 1 and 3 months after hospital discharge, respectively. Of the discharged patients, $18(18.6 \%)$ and $28(28.9 \%)$ had an unplanned ED readmission within 1 and 3 months after ED discharge, respectively. The fTRST, Rowland, and GUGT were not completed for 11 (2.9\%), 14 (3.7\%), and 23 $(6.0 \%)$ patients, respectively.

\section{Prediction of unplanned ED readmission in hospitalized and discharged patients}

In both hospitalized and discharged patients, the fTRST and Rowland using a cut-off at least 2 showed good to excellent sensitivity and NPV, but low to moderate specificity and accuracy. The GUGT showed low sensitivity and good to excellent NPV and specificity. The
AUC was low to moderate and ranged between 0.48 and 0.64 for all instruments and all cut-offs (Supplementary data 2, Supplemental digital content 2, http://links.lww. com/EJEM/A127, Supplemental digital content 3, http:// links.lww.com/EJEM/A128).

In hospitalized patients, combining the fTRST or Rowland with the GUGT showed good to excellent NPV (71.4-94.3\%), with the highest NPV observed at the 1-month follow-up. When combining the fTRST and GUGT, sensitivity was low, with no significant changes when the original cut-off of at least 2 was increased or decreased. By contrast, specificity was moderate $(61.9-71.6 \%)$, and it increased when using a cut-off at least 1 . Sensitivity was good $(80.0 \%)$ at 1 month when combining the Rowland (cut-off $\geq 4$ ) and GUGT, but decreased (56.5\%) at the 3-month follow-up. Specificity, however, was low (46.3-49.3\%) and increased slightly when applying lower cut-offs. Decreasing the cut-off of the Rowland to at least 2 resulted in a decrease in sensitivity. Overall, the AUC was moderate and ranged between 0.51 and 0.65 (Table 2).

In discharged patients, combining the fTRST or Rowland with the GUGT produced a somewhat lower NPV (56.3-85.2\%) compared with that of the hospitalized group, with the highest the NPVs observed at the 1-month follow-up. When combining the fTRST and GUGT, sensitivity was very low, with no significant improvements when increasing or decreasing the original cut-off of at least 2, whereas the specificity was good to very good (88.9-93.2\%). The sensitivity of the combined Rowland (cut-off $\geq 4$ )/GUGT was low (11.1-40.0\%). Decreasing the Rowland/GUGT cut-off to at least 2 also resulted in a decrease in sensitivity. Specificity, in contrast, was good to very good (81.8-92.0\%) and increased slightly on lowering the cut-off. Overall, the AUC was moderate and ranged between 0.49 and 0.63 (Table 3).

\section{Discussion}

This study evaluated the diagnostic characteristics of the GUGT when used as a stand-alone test and when used in combination with existing screening instruments (i.e. fTRST, Rowland questionnaire). The main aim was to examine the efficacy of these tools for predicting unplanned ED readmission in patients aged 75 years or older. To the best of our knowledge, this was the first study to evaluate these tests using a two-step approach, including a traditional screening instrument and the GUGT.

The GUGT evaluates patients' global physical activity and is different from traditional screening instruments tested previously in an ED setting. Despite the recommendation of Carpenter et al. [18] to add objective assessments to improve accuracy, our results show that the GUGT used as a stand-alone test is not sufficiently sensitive to predict unplanned ED readmissions. This 


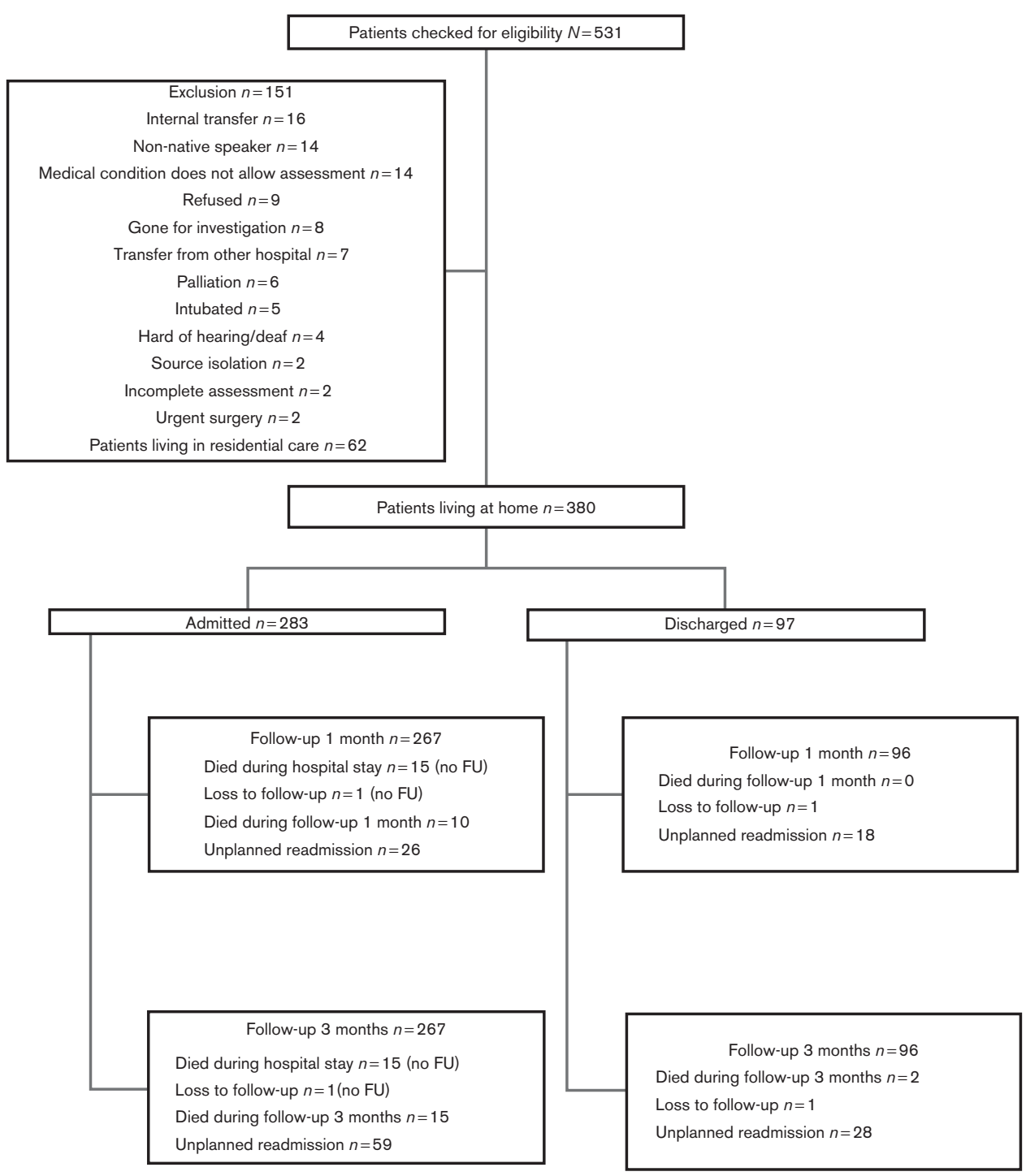

Flow chart schematic for patient selection and categorization.

low sensitivity results in the underdetection of patients at risk; in other words, it leads to false negatives.

Combining both patient self-reported responses and objective assessment of mobility by adding the GUGT to the fTRST or the Rowland questionnaire failed to improve the diagnostic accuracy of the latter instruments. It appears that adding the GUGT as an objective measurement may not be a solution to overcoming the shortcomings of the fTRST and Rowland. However, from a clinical point of view, the GUGT can still be used as a test to provide important information for discharge decisions in patients who are unable to stand up and walk independently.

Despite the small methodological differences in terms of the selected sample and time frame, this study confirms earlier findings on the stand-alone tests by showing high sensitivity, low specificity, and high NPV as a stand-alone test $[15,17]$. The high number of false-positive detections in our study implies that these instruments should not be used as a stand-alone test, but should instead be used as a first step to safely exclude patients who would not benefit from further geriatric evaluation.

The sensitivity and NPV decreased over time (1 vs. 3 months) for both the stand-alone tests and the two-step approach, indicating that long-term unplanned ED readmission is more difficult to predict than short-term readmissions. This is likely because of the rapidly changing medical condition of frail older patients and readmissions that are unrelated to the initial reason for ED admission. Similar results for predictive accuracy 
Table 1 Patient characteristics stratified by type

\begin{tabular}{|c|c|c|c|}
\hline Characteristics & $\begin{array}{l}\text { Total sample } \\
(n=380)\end{array}$ & $\begin{array}{l}\text { Hospitalized } \\
(n=283)\end{array}$ & $\begin{array}{l}\text { Discharged } \\
\quad(n=97)\end{array}$ \\
\hline Age $[$ mean $( \pm S D)]$ & $83.1( \pm 4.5)$ & $83.2( \pm 4.4)$ & $82.54( \pm 4.8)$ \\
\hline Female $[n(\%)]$ & $224(58.9)$ & $161(56.9)$ & $63(64.9)$ \\
\hline \multicolumn{4}{|l|}{ Living situation $[n(\%)]$} \\
\hline Home, alone & $155(40.8)$ & $118(41.7)$ & $37(38.1)$ \\
\hline $\begin{array}{l}\text { Home, together with } \\
\text { relative }\end{array}$ & $225(59.2)$ & $165(58.3)$ & $60(61.9)$ \\
\hline $\begin{array}{l}\text { Received nursing care } \\
\text { at home }[n(\%)]\end{array}$ & $121(32.4)$ & $96(34.4)$ & $25(26.3)$ \\
\hline $\begin{array}{l}\text { Received home care } \\
{[n(\%)]}\end{array}$ & $48(12.8)$ & $41(14.7)$ & $7(7.3)$ \\
\hline $\begin{array}{l}\text { Received meals on } \\
\text { wheels }[n(\%)]\end{array}$ & $58(15.5)$ & $49(17.6)$ & $9(9.4)$ \\
\hline $\begin{array}{l}\text { Received cleaning help } \\
{[n(\%)]}\end{array}$ & $170(45.3)$ & $126(45.2)$ & $44(45.8)$ \\
\hline $\begin{array}{l}\text { Received shopping } \\
\text { assistance }[n(\%)]\end{array}$ & $238(63.6)$ & $187(66.1)$ & $51(52.6)$ \\
\hline $\begin{array}{l}\text { Falls in the last year } \\
{[n(\%)]}\end{array}$ & $202(53.2)$ & $150(53.0)$ & $52(53.6)$ \\
\hline $\begin{array}{l}\text { ADL premorbid } \\
\text { [median (Q1-Q3)] }\end{array}$ & $6.0(6-8)$ & $6.5(6-9)$ & $6.0(6-7)$ \\
\hline $\begin{array}{l}\text { Cognitively impaired } \\
{[n(\%)]}\end{array}$ & $82(21.6)$ & $70(24.7)$ & $12(12.4)$ \\
\hline Had delirium $[n(\%)]$ & $24(6.5)$ & $23(8.1)$ & $1(1.0)$ \\
\hline CIRS [mean $( \pm \mathrm{SD})]$ & $13.4( \pm 4.7)$ & $13.9( \pm 4.6)$ & $11.61( \pm 4.805)$ \\
\hline \multicolumn{4}{|l|}{$\operatorname{ESI}[n(\%)]^{a}$} \\
\hline 1 & $1(0.3)$ & $1(0.4)$ & $0(0.0)$ \\
\hline 2 & $128(38.8)$ & $96(39.3)$ & $32(37.2)$ \\
\hline 3 & $175(53.0)$ & $134(55.0)$ & $41(47.7)$ \\
\hline 4 & $25(7.6)$ & $12(4.9)$ & $13(15.1)$ \\
\hline 5 & $1(0.3)$ & $1(0.4)$ & $0(0.0)$ \\
\hline $\begin{array}{l}\text { Length of stay in hours } \\
\text { and minutes, median }\end{array}$ & $\begin{array}{l}15 \mathrm{~h} 57 \mathrm{~min} \\
\quad(7 \mathrm{~h}\end{array}$ & $\begin{array}{l}13 \mathrm{~h} 24 \mathrm{~min}(8 \mathrm{~h} \\
40 \mathrm{~min}-1 \text { day }\end{array}$ & $\begin{array}{c}8 \mathrm{~h} 22 \min (5 \mathrm{~h} \\
42 \mathrm{~min}-16 \mathrm{~h}\end{array}$ \\
\hline (Q1-Q3) & $\begin{array}{l}59 \mathrm{~min}-23 \mathrm{~h} \\
42 \mathrm{~min})\end{array}$ & $1 \mathrm{~h} 15 \mathrm{~min})$ & $37 \mathrm{~min})$ \\
\hline \multicolumn{4}{|c|}{ Primary diagnosis at discharge $[n(\%)]$} \\
\hline General decline & $145(38.2)$ & $115(40.6)$ & $30(30.9)$ \\
\hline Trauma & $30(7.9)$ & $18(6.4)$ & $12(12.4)$ \\
\hline Cardiovascular & $96(25.3)$ & $12(4.2)$ & $7(7.2)$ \\
\hline Neurological & $19(5.0)$ & 66 (23.3) & $30(31.0)$ \\
\hline Gastrointestinal & $30(8.0)$ & $29(10.2)$ & $1(1.0)$ \\
\hline Respiratory & $13(3.4)$ & $9(3.2)$ & $4(4.1)$ \\
\hline Oncological & $4(1.1)$ & $4(1.4)$ & $0(0.0)$ \\
\hline Kidney/urological & $13(3.4)$ & $9(3.2)$ & $4(4.1)$ \\
\hline Other & $23(6.1)$ & $17(6.0)$ & $6(6.2)$ \\
\hline No firm diagnosis & $7(1.8)$ & $4(1.4)$ & (3.1) \\
\hline
\end{tabular}

ADL, activities of daily living; CIRS, Modified Cumulative Illness Rating Scale; ESI, Emergency Severity Index.

${ }^{\text {aT There were }} 50$ missing data values. ESI not given during night-time. were observed in both hospitalized and discharged patients, suggesting no need for the use of different screening instruments for these groups.

The present study shows that neither the stand-alone GUGT nor the combination of fTRST and Rowland with the GUGT provides accurate predictions of unplanned readmission. However, the results of using the fTRST or the Rowland questionnaire (cut-off $=2$ ) as a stand-alone test are much better, taking into account the known and previously mentioned limitations. Geriatric screening using fTRST or Rowland questionnaire can be used in the process of clinical evaluation in the ED. First, use of the instrument will increase the alertness of healthcare workers in the ED for geriatric syndromes or patients with a geriatric risk profile. Second, one solution to or compensation for the high rate of false-positive cases may be the integration of the "clinical impression that further assessment is needed to prevent adverse health outcomes' of the healthcare workers as a second step following geriatric screening. Third, risk screening should be seen as one of the first steps in a more global process. An additional ED-adapted geriatric assessment can help to confirm or negate the results of the screening, highlight specific problems (e.g. cognitive impairment), and help to map the subsequent trajectory of an older individual after an ED visit and the medical diagnosis process are completed. Of course, the local context of the ED plays an important role in the timing of additional assessment, which can be executed during the ED visit, during the hospital stay, or at home after discharge.

Fourth, an alternative for traditional screening instruments such as fTRST and Rowland could be the new third generation of Resident Assessment Instruments (InterRAI) that are introduced within geriatric care. The InterRAI ED screener is based on an algorithm and predicts the need for follow-up assessment and the risk of poor outcomes [28,29]. Finally, other objective parameters in other domains - e.g., screening for cognitive

Table 2 Predictive values when using a combination of fTRST or Rowland with GUGT in hospitalized patients 1 and 3 months after discharge

\begin{tabular}{|c|c|c|c|c|c|c|c|c|c|}
\hline Tool & Cut-off & Sensitivity (\%) & Specificity $(\%)$ & PPV (\%) & NPV (\%) & $\mathrm{LH}+(\%)$ & $\mathrm{LH}-(\%)$ & AUC (\%) & Accuracy (\%) \\
\hline \multicolumn{10}{|c|}{1 month after discharge } \\
\hline \multirow[t]{3}{*}{ fTRST + GUGT } & $1(n=234)$ & 44.0 & 71.3 & 15.5 & 91.4 & 1.533 & 0.786 & 0.576 & 68.4 \\
\hline & $2(n=186)$ & 45.8 & 64.8 & 16.2 & 89.0 & 1.303 & 0.836 & 0.553 & 62.4 \\
\hline & $3(n=117)$ & 47.1 & 63.0 & 17.8 & 87.5 & 1.272 & 0.840 & 0.550 & 60.7 \\
\hline \multirow[t]{3}{*}{ Rowland + GUGT } & $2(n=152)$ & 43.5 & 62.8 & 17.2 & 86.2 & 1.168 & 0.900 & 0.531 & 59.9 \\
\hline & $3(n=108)$ & 60.0 & 58.1 & 18.8 & 90.0 & 1.431 & 0.689 & 0.590 & 58.3 \\
\hline & $4(n=77)$ & 80.0 & 49.3 & 19.0 & 94.3 & 1.576 & 0.406 & 0.646 & 53.2 \\
\hline \multicolumn{10}{|c|}{3 months after discharge } \\
\hline \multirow[t]{3}{*}{ fTRST + GUGT } & $1(n=234)$ & 37.3 & 71.6 & 26.8 & 80.4 & 1.311 & 0.877 & 0.544 & 64.1 \\
\hline & $2(n=186)$ & 39.6 & 64.5 & 27.9 & 75.4 & 1.115 & 0.937 & 0.520 & 58.1 \\
\hline & $3(n=117)$ & 39.4 & 61.9 & 28.9 & 72.2 & 1.034 & 0.979 & 0.506 & 55.6 \\
\hline \multirow[t]{3}{*}{ Rowland + GUGT } & $2(n=152)$ & 44.2 & 64.2 & 32.8 & 74.5 & 1.235 & 0.869 & 0.542 & 58.6 \\
\hline & $3(n=108)$ & 48.4 & 57.1 & 31.3 & 73.3 & 1.129 & 0.903 & 0.528 & 54.6 \\
\hline & $4(n=77)$ & 56.5 & 46.3 & 31.0 & 71.4 & 1.052 & 0.939 & 0.514 & 49.4 \\
\hline
\end{tabular}

AUC, area under the curve; fTRST, Flemish Triage Risk Screening Tool; GUGT, get up and go test; LH-, likelihood ratio of negative test; LH+, likelihood ratio of positive test; NPV, negative predictive value; PPV, positive predictive value. 
Table 3 Predictive values of the combination of fTRST or Rowland with GUGT in the group of discharged patients 1 and 3 months after discharge $\mathrm{e}^{\mathrm{a}}$

\begin{tabular}{|c|c|c|c|c|c|c|c|c|c|}
\hline Tool & Cut-off & Sensitivity (\%) & Specificity (\%) & PPV (\%) & NPV (\%) & $\mathrm{LH}+(\%)$ & $\mathrm{LH}-(\%)$ & AUC (\%) & Accuracy $(\%)$ \\
\hline \multicolumn{10}{|c|}{1 month after discharge } \\
\hline \multirow[t]{3}{*}{ fTRST + GUGT } & $1(n=78)$ & 12.5 & 91.9 & 28.6 & 80.3 & 1.550 & 0.952 & 0.522 & 75.6 \\
\hline & $2(n=57)$ & 15.4 & 93.2 & 40.0 & 78.8 & 2.256 & 0.908 & 0.543 & 75.4 \\
\hline & $3(n=31)$ & 25.0 & 91.3 & 50.0 & 77.8 & 2.875 & 0.825 & 0.582 & 74.2 \\
\hline \multirow[t]{3}{*}{ Rowland + GUGT } & $2(n=47)$ & 18.2 & 91.7 & 40.0 & 78.6 & 2.182 & 0.893 & 0.549 & 74.5 \\
\hline & $3(n=31)$ & 33.3 & 92.0 & 50.0 & 85.2 & 4.167 & 0.725 & 0.627 & 80.6 \\
\hline & $4(n=20)$ & 40.0 & 86.7 & 50.0 & 81.3 & 3.000 & 0.692 & 0.633 & 75.0 \\
\hline \multicolumn{10}{|c|}{3 months after discharge } \\
\hline \multirow[t]{3}{*}{ FTRST + GUGT } & $1(n=78)$ & 8.0 & 90.6 & 28.6 & 67.6 & 0.848 & 1.016 & 0.493 & 64.1 \\
\hline & $2(n=57)$ & 9.1 & 91.4 & 40.0 & 61.5 & 1.061 & 0.994 & 0.503 & 59.6 \\
\hline & $3(n=31)$ & 15.4 & 88.9 & 50.0 & 59.3 & 1.385 & 0.952 & 0.521 & 58.1 \\
\hline \multirow[t]{3}{*}{ Rowland + GUGT } & $2(n=47)$ & 11.1 & 89.7 & 40.0 & 61.9 & 1.074 & 0.991 & 0.504 & 59.6 \\
\hline & $3(n=31)$ & 18.2 & 90.0 & 50.0 & 66.7 & 1.818 & 0.909 & 0.541 & 64.5 \\
\hline & $4(n=20)$ & 22.2 & 81.8 & 50.0 & 56.3 & 1.22 & 0.951 & 0.520 & 55.0 \\
\hline
\end{tabular}

AUC, area under the curve; fTRST, Flemish Triage Risk Screening Tool; GUGT, get up and go test; LH-, likelihood ratio of negative test; LH +, likelihood ratio of positive test; NPV, negative predictive value; PPV, positive predictive value.

${ }^{\text {a }}$ Same conventions as Table 2.

decline combined with the traditional screenings instruments - need to be evaluated to test the hypotheses stated in a recent meta-analysis [18].

The majority of older patients in the ED are admitted to the hospital after an ED visit. This may be related to the fact that the setting for this study was a university hospital with a level I trauma center. This possibly could result in a more complex profile of patients needing more hospitalizations.

Within 3 months of the index visit, almost one-third of the discharged patients and one out of five of the hospitalized patients were readmitted to the hospital. These numbers are comparable with those found in previous studies [6,7], underscoring the need for early identification of older patients at risk for readmission. Hence, proactive assessment and healthcare interventions can be performed during and after the ED stay.

The following methodological limitations should be taken into account when interpreting the study results. First, the group of discharged patients was small, increasing the risk for selection bias and studying an unrepresentative sample of the population. However, the incidence of unplanned ED readmission in this subgroup was comparable with that found in previous studies, arguing against this possibility. Second, unplanned readmissions in the hospitalized group were assessed using only chart review, meaning that there could have been unplanned ED readmissions outside of the study hospital that were not recorded. However, it is reasonable to assume that older patients tend to visit the same hospital, and thus this possibility may be less likely. Third, there were some missing data in the screeners, but the overall percentage per instrument was rather low (between 2.9 and 6\%). There were somewhat more missing data in the GUGT results because the test could not always be performed, for example, patients with mandatory bed rest, fractures, or those receiving oxygen could not be tested. Fourth, although the GUGT is an easy-to-perform test in the ED, there were still some situations in which the test was not performed in the way it was validated. The need for intensive monitoring while executing the test and infrastructural limitations in the ED sometimes precluded a walk as far as three meters. Next, although all research nurses were trained before the start of the study to improve scoring consistency, we did not evaluate inter-rater and intra-rater reliability; thus, increased variability in scoring among the nurses would have decreased statistical power, if present. Finally, this study evaluated unplanned, but not preventable ED readmissions, which means that the readmission rates included patients for whom a revisit to the ED is legitimate because of an urgent medical problem.

\section{Conclusion}

The results of the predictive accuracy of the stand-alone self-reported screening instruments fTRST and Rowland questionnaire (cut-off $=2$ ) are good, and the previously known limitations were confirmed in this study. Neither the objective assessment of mobility (GUGT) nor the combination of the GUGT with the fTRST or Rowland questionnaire improved predictive accuracy. Since the group of older people on the ED is still growing, a simple and quick identification of vulnerable geriatric patients remains a critical first step in the process of clinical evaluation during the ED visit. This early identification can lead to an adapted care trajectory in which continuity of care is warranted.

\section{Acknowledgements}

The authors gratefully acknowledge the staff of the ED at the University Hospitals Leuven and all consenting participants. They also thank the research assistants Anne Jamaer and Nicky Hasevoets. The authors were supported by a grant from the Borgerhoff Fund 2012, Belgian Society for Gerontology and Geriatrics, Belgium. 


\section{Conflicts of interest}

There are no conflicts of interest.

\section{References}

1 Aminzadeh F, Dalziel WB. Older adults in the emergency department: a systematic review of patterns of use, adverse outcomes, and effectiveness of interventions. Ann Emerg Med 2002; 39:238-247.

2 Gruneir A, Silver MJ, Rochon PA. Emergency department use by older adults: a literature review on trends, appropriateness, and consequences of unmet health care needs. Med Care Res Rev 2011; 68:131-155.

3 Lowenstein SR, Crescenzi CA, Kern DC, Steel K. Care of the elderly in the emergency department. Ann Emerg Med 1986; 15:528-535.

4 Deschodt M, Devriendt E, Sabbe M, Knockaert D, Deboutte P, Boonen S, et al. Characteristics of older adults admitted to the emergency department (ED) and their risk factors for ED readmission based on comprehensive geriatric assessment: a prospective cohort study. BMC Geriatr 2015; 15:54.

5 McCusker J, Cardin S, Bellavance F, Belzile E. Return to the emergency department among elders: patterns and predictors. Acad Emerg Med 2000; 7:249-259.

6 Moons P, de Ridder K, Geyskens K, Sabbe M, Braes T, Flamaing J, et al. Screening for risk of readmission of patients aged 65 years and above after discharge from the emergency department: predictive value of four instruments. Eur J Emerg Med 2007; 14:315-323.

7 Braes T, Moons P, Lipkens P, Sterckx W, Sabbe M, Flamaing J, et al. Screening for risk of unplanned readmission in older patients admitted to hospital: predictive accuracy of three instruments. Aging Clin Exp Res 2010; 22:345-351.

8 Rubenstein LZ, Stuck AE, Siu AL, Wieland D. Impacts of geriatric evaluation and management programs on defined outcomes: overview of the evidence. J Am Geriatr Soc 1991; 39 (Pt 2):8S-16S.

9 Sinha SK, Bessman ES, Flomenbaum N, Leff B. A systematic review and qualitative analysis to inform the development of a new emergency department-based geriatric case management model. Ann Emerg Med 2011; 57:672-682.

10 Foo CL, Siu VW, Tan TL, Ding YY, Seow E. Geriatric assessment and intervention in an emergency department observation unit reduced reattendance and hospitalisation rates. Australas J Ageing 2012; 31:40-46.

11 Arendts G, Fitzhardinge S, Pronk K, Donaldson M, Hutton M, Nagree Y. The impact of early emergency department allied health intervention on admission rates in older people: a non-randomized clinical study. BMC Geriatr 2012; 12:8.

12 Wright PN, Tan G, lliffe S, Lee D. The impact of a new emergency admission avoidance system for older people on length of stay and same-day discharges. Age Ageing 2014; 43:116-121.

13 Conroy SP, Stevens T, Parker SG, Gladman JR. A systematic review of comprehensive geriatric assessment to improve outcomes for frail older people being rapidly discharged from acute hospital: 'interface geriatrics'. Age Ageing 2011; 40:436-443.

14 Graf CE, Zekry D, Giannelli S, Michel JP, Chevalley T. Efficiency and applicability of comprehensive geriatric assessment in the emergency department: a systematic review. Aging Clin Exp Res 2011; 23:244-254.
15 Buurman BM, van den Berg W, Korevaar JC, Milisen K, de Haan RJ, de Rooij SE. Risk for poor outcomes in older patients discharged from an emergency department: feasibility of four screening instruments. Eur $J$ Emerg Med 2011; 18:215-220.

16 Salvi F, Morichi V, Lorenzetti B, Rossi L, Spazzafumo L, Luzi R, et al. Risk stratification of older patients in the emergency department: comparison between the identification of seniors at risk and triage risk screening tool. Rejuvenation Res 2012; 15:288-294.

17 Graf CE, Giannelli SV, Herrmann FR, Sarasin FP, Michel JP, Zekry D, et al. Identification of older patients at risk of unplanned readmission after discharge from the emergency department comparison of two screening tools. Swiss Med Wkly 2012; 141 :w13327.

18 Carpenter CR, Shelton E, Fowler S, Suffoletto B, Platts-Mills TF, Rothman RE, et al. Risk factors and screening instruments to predict adverse outcomes for undifferentiated older emergency department patients: a systematic review and meta-analysis. Acad Emerg Med 2015; 22:1-21.

19 Gine-Garriga M, Guerra M, Mari-Dell'Olmo M, Martin C, Unnithan VB. Sensitivity of a modified version of the 'timed get up and go' test to predict fall risk in the elderly: a pilot study. Arch Gerontol Geriatr 2009; 49:e60-e66.

20 Walker KJ, Bailey M, Bradshaw SJ, Cameron P, Dziukas L, Maguire EK, et al. Timed up and go test is not useful as a discharge risk screening tool. Emerg Med Australas 2006; 18:31-36.

21 Tanabe P, Gimbel R, Yarnold PR, Kyriacou DN, Adams JG. Reliability and validity of scores on the emergency severity index version 3. Acad Emerg Med 2004; 11:59-65.

22 Salvi F, Miller MD, Grilli A, Giorgi R, Towers AL, Morichi V, et al. A manual of guidelines to score the modified cumulative illness rating scale and its validation in acute hospitalized elderly patients. J Am Geriatr Soc 2008; 56:1926-1931

23 Inouye SK, van Dyck CH, Alessi CA, Balkin S, Siegal AP, Horwitz RI. Clarifying confusion: the confusion assessment method. A new method for detection of delirium. Ann Intern Med 1990; 113:941-948.

24 Fong TG, Jones RN, Rudolph JL, Yang FM, Tommet D, Habtemariam D, et al. Development and validation of a brief cognitive assessment tool: the sweet 16. Arch Intern Med 2011; 171:432-437.

25 Katz S, Ford AB, Moskowitz RW, Jackson BA, Jaffe MW. Studies of illness in the aged. The index of ADL: a standardized measure of biological and psychosocial function. Jama 1963; 185:914-919.

26 Landrum L, Weinrich S. Readmission data for outcomes measurement: identifying and strengthening the empirical base. Qual Manag Health Care 2006; 15:83-95.

27 Rowland K, Maitra AK, Richardson DA, Hudson K, Woodhouse KW. The discharge of elderly patients from an accident and emergency department: functional changes and risk of readmission. Age Ageing 1990; 19:415-418

28 Hirdes J, Curtin-Telegdi N, Poss J, Gray L, Berg K, Stolee P, et al. interRAI Contact Assessment (CA) form and user's manual: a screening level assessment for Emergency Department and intake from community/ hospital, version 9.2. Washington, DC: interRAl; 2010.

29 Ontario Hospital Association. Release of the interRAl Emergency Department (ED) Screener [Internet]. Toronto, Ontario (Canada): Ontario Hospital Association; 2014. Available at: http://www.healthscape.ca/Pages/ resources-06052014-mhealthinterraiapp.aspx. [Accessed 27 December 2015]. 